\title{
Review of: "Rationale and Design of the Vasospastic Angina Treatment by Endothelin Receptor Antagonism (VERA) Trial"
}

Hiroki Teragawa

Potential competing interests: The author(s) declared that no potential competing interests exist.

This was a position paper on the VERA trial of endothelin receptor antagonist in patients with vasospastic angina (VSA) or microvascular angina (MVA), and I think it was well written.

\#1 The protocol of present study has already been presented, and it may be difficult to change it now, but I think that the frequency or number of nitroglycerin use could have been included in the secondary evaluation.

\#2 What proportion of VSA and MVA is expected to be included? In general, I think that VSA patients are more likely to respond to drugs, and MVA patients are drug resistant, and I think that the cases to be recruited will vary depending on the frequency of these diseases. 